Europhysics Letters

PREPRINT

\title{
Continuous optical loading of a Bose-Einstein condensate in the Thomas-Fermi regime
}

\author{
F. Floegel, L. Santos And M. Lewenstein \\ Institut für Theoretische Physik, Universität Hannover, D-30167 Hannover, Germany \\ PACS. 42.50.Vk - Mechanical effects of light on atoms. \\ PACS. 03.75.Kk - Dynamic properties of condensates; collective and hydrodynamic excita- \\ tions,superfluid flow. \\ PACS. 03.75.Pp - Atom lasers.
}

\begin{abstract}
We discuss the optical loading of a Bose-Einstein condensate in the Thomas-Fermi regime. The condensate is loaded via spontaneous emission from a reservoir of excited-state atoms. By means of a master equation formalism, we discuss the modification of the condensate temperature during the loading. We identify the threshold temperature, $T_{t h}$, above (below) which the loading process leads to cooling (heating), respectively. The consequences of our analysis for the continuous loading of an atom laser are discussed.
\end{abstract}

The experimental realization of a Bose-Einstein condensate (BEC) has aroused an enormous interest during the recent years [1. Among the disparate variety of topics related with the BEC physics, the possibility of accomplishing a coherent source of atoms, or atom laser, has attracted a large attention [2]. As a coherent source of matter waves, the atom laser is expected to lead to new applications in atom optics, and its relevance is often compared to the impact of optical lasers in standard optics. The atom-laser has been produced experimentally after outcoupling atoms from a BEC either by employing radio-frequency fields [3, 4, or Raman techniques [5]. The coherent character of the source has been demonstrated in a series of interferometric experiments [4, 6, 7. The availability of continuous-wave (cw) atom lasers would open the way to "high power" and precision applications. Several groups have developed various techniques to outcouple atoms in a (quasi-) continuous way [5]4. However, the continuous outcoupling represents just a half way towards a cw atom laser. Without a continuous refilling of the BEC, the atom laser output lasts only as long as some atoms in the BEC are kept. In addition, the continuous refilling of the condensate could be employed to repair a condensate against inelastic losses, and in this way it could allow to analyze the BEC physics at larger densities, at much longer time scales. Recently, the first steps toward this direction has been achieved in an experiment performed at MIT 8 .

Two different physical mechanisms could provide a continuous pumping into a BEC. On one hand, collisional mechanisms [9, in which two non-condensed reservoir atoms collide, one being pumped into the BEC, whereas the other carries most of the energy and is evaporated. Several proposals have been suggested in this direction. Ref. [9] analyzes the continuous evaporative cooling of an atomic beam directed along a long magnetic guide, at the end of

(C) EDP Sciences 
which the atoms are predicted to enter the degenerate regime. This method demands a large flux of slow atoms in order to achieve a sufficiently large collisional rate which guarantees the required efficiency of the evaporative cooling in a finite guide. Recently, Roos et al. [10] have analyzed a scheme in which particles from an incident beam are trapped in a potential well when colliding with particles already present in the well. For a cigar-shape potential, it has been shown that a resonance can occur when the transverse evaporation threshold coincides with the energy of the incident particles, leading to a large increase in phase space density with respect to the incident beam. We should also refer at this point to a recent experiment [8], where, by means of an optical tweezer, a BEC is merged with a pre-existing one. A second way of continuous loading of a BEC is provided by the spontaneous emission from a reservoir of excited-state atoms 11,12 13. If this reservoir could be filled in a (quasi-) continuous way by laser cooling techniques, one would benefit from the large cooling efficiency of laser cooling compared to evaporative cooling, allowing for a considerable increase in atomic flux produced by an atom laser. In order to achieve the continuous optical loading of a BEC, it is crucial to avoid the heating introduced by the photon reabsorption [14. Fortunately, it has been shown that in the so-called Festina Lente regime, in which the spontaneous emission rate, $\gamma$, is smaller than the frequency of the harmonic trapping, $\omega$, the heating due to reabsorption is practically suppressed [15]. It has been also recently reported that, under certain conditions, the reabsorption can be also largely suppressed in atoms with an accessible three level $\Lambda$ scheme [12. Additionally, quantum interference effects can lead to positive (cooling) effects of the reabsorption in the so-called BAR regime [16.17.

The continuous optical loading of a BEC have been previously analyzed in ref. 12, in which the $\mathrm{BEC}$ was considered in the weak condensation regime, where the mean interaction energy is smaller than the separation between the harmonic levels. In that case, the loading dynamics can be split from the collisional physics in the corresponding quantum Master equation (QME), and both phenomena can be simultaneously simulated via Monte Carlo methods [18, 12. In particular in ref. 12], it was shown that a BEC can be first created in an initially empty trap by means of optical loading, and then maintained against losses by properly fixing the pumping rate. However, the weakly-interacting regime sets important restrictions to the atomic density. In the present paper, we extend our analysis to arbitrary regimes, and in particular to the Thomas-Fermi regime, in which the mean interaction energy is much larger than the separation between the trap levels. In that case, the BEC wavefunction and the excitations depend on the particle number, and as consequence the wavefunctions vary dynamically during the pumping process, complicating the analysis of the problem. In this paper we develop the necessary theory to analyze the problem. In particular, we study the modification of the condensate temperature during the pumping process. We show that there is a threshold temperature for a given number of trapped particles above (below) which the system is cooled (heated), and show that this effect could be employed to control the condensate temperature.

We consider a sample of cold atoms of mass $m$ with an accessible electronic two-level system, formed by the ground state $|g\rangle$ and an excited state $|e\rangle$. Both $|g\rangle$ and $|e\rangle$ atoms are in a harmonic trap, which for simplicity is considered isotropic, with frequencies $\omega_{g}$ and $\omega_{e}$, respectively. The $|g\rangle$ atoms are Bose condensed, i.e. with a temperature $T \ll T_{c}$, where $T_{c}$ is the critical temperature for onset of the condensation. The formation of the condensate using spontaneous emission was previously discussed in ref. 12. The $|e\rangle$ atoms, which eventually decay spontaneously into the $|g\rangle$ state, are considered as thermally distributed.

The atoms in the $|g\rangle$ trap are described by the corresponding field operator $\hat{\Psi}_{g}(\vec{r})$. For sufficiently low $T$, when the number of condensed atoms in the $|g\rangle$ trap, $N_{0}$, is comparable to the total number of atoms, $N$, one can employ the Bogoliubov approximation and substitute $\hat{\Psi}_{g}$ by a $c$-number, $\psi_{0}$, the BEC wave function, whose stationary value is provided by the 
time-independent Gross-Pitaevskii equation (GPE)

$$
\mathcal{H}_{G P} \psi_{0}(\vec{r})=\left(-\frac{\hbar^{2}}{2 m} \nabla^{2}+\frac{m}{2} \omega_{g}^{2} r^{2}+U_{g g} N\left|\psi_{0}\right|^{2}-\mu\right) \psi_{0}(\vec{r})=0
$$

where $\mu$ is the chemical potential, and $U_{g g}=4 \pi \hbar^{2} a_{g g} / m$ is the coupling constant for the collisions between $|g\rangle$ atoms, with $a_{g g}$ the scattering length. In eq. (1) we have neglected the $e-g$ collisions, since the number of atoms in the $|e\rangle$ state is assumed very small.

The excitation spectrum is obtained after linearizing $\hat{\Psi}_{g}$ around the ground-state solution, $\hat{\Psi}_{g}(\vec{r})=\psi_{0}(\vec{r})+\delta \hat{\psi}(\vec{r})$. The perturbation $\delta \hat{\psi}$ is then expanded in the standard form [19] $\delta \hat{\psi}(\vec{r})=u_{\vec{n}}^{*}(\vec{r}) \tilde{g}_{\vec{n}}-v_{\vec{n}}(\vec{r}) \tilde{g}_{\vec{n}}^{\dagger}$, where $\tilde{g}_{\vec{n}}$ and $\tilde{g}_{\vec{n}}^{\dagger}$ are the annihilation and creation operators for the quasiparticles with spherical quantum numbers $\vec{n}=n, l, m$. The wave functions $u_{\vec{n}}(\vec{r})$ and $v_{\vec{n}}(\vec{r})$ obey the standard orthonormalization rules [19]. Linearizing in the $u_{\vec{n}}(\vec{r})$ and $v_{\vec{n}}(\vec{r})$ wavefunctions, one obtains the corresponding Bogoliubov equations

$$
\begin{aligned}
& \left(\mathcal{H}_{G P}+U_{g g} N_{0} Q \psi_{0}^{2} Q\right) u_{\vec{n}}+U_{g g} N_{0} Q \psi_{0}^{2} Q^{*} v_{\vec{n}}=\hbar \tilde{\omega}_{\vec{n}}^{g} u_{\vec{n}} \\
& -U_{g g} N_{0} Q^{*} \psi_{0}(\vec{r})^{2} Q u_{\vec{n}}-\left(\mathcal{H}_{G P}+U_{g g} N_{0} Q \psi_{0}^{2} Q\right)^{*} v_{\vec{n}}=\hbar \tilde{\omega}_{\vec{n}}^{g} v_{\vec{n}}
\end{aligned}
$$

where we have used the projection operators $Q=1-\left|\psi_{0}\right\rangle\left\langle\psi_{0}\right|\left(Q^{*}\right)$ orthogonally to $\psi_{0}$ $\left(\psi_{0}^{*}\right)$ [21], and $\tilde{\omega}_{\vec{n}}^{g}$ denotes the quasiparticle excitation spectrum.

The physics of the atoms in the $|e\rangle$ state is described by the Schrödinger equation:

$$
\mathcal{H}_{e} \psi_{\vec{m}}^{e}(\vec{r})=\left(-\frac{\hbar^{2}}{2 m} \nabla^{2}+\frac{m}{2} \omega_{e}^{2} r^{2}+2 U_{g e} N_{0}\left|\psi_{0}\right|^{2}\right) \psi_{\vec{m}}^{e}(\vec{r})=\hbar \tilde{\omega}_{\vec{m}}^{e} \psi_{\vec{m}}^{e}(\vec{r}),
$$

where we have taken into account that the $|e\rangle$ atoms are affected by the mean-field potential induced by the collisions with the $|g\rangle$ atoms, which are characterized by a coupling constant $U_{g e}=4 \pi \hbar^{2} a_{g e} / m$, with $a_{g e}$ the corresponding scattering length. Due to the low density in the $|e\rangle$ trap, we have neglected in eq. (4) the $e$-e collisions. In the following, $\tilde{e}_{\vec{m}}^{\dagger}$ and $\tilde{e}_{\vec{m}}$ denote the creation and annihilation operators in the eigenstate $\psi_{\vec{m}}^{e}$, with eigenfrequency $\tilde{\omega}_{\vec{m}}^{e}$.

The interaction of the atoms with the vacuum electromagnetic field is given by

$$
\mathcal{H}_{a f}=i \sum_{\vec{m}} \sum_{v} \int d \vec{k} \rho(\vec{k})\left(\vec{d}_{\epsilon_{k v}}\right)\left(\eta_{0 \vec{m}}^{c} \tilde{g}_{0}^{\dagger} e_{\vec{m}}+\sum_{\vec{n}} \eta_{\vec{n} \vec{m}}^{u} \tilde{g}_{\vec{n}}^{\dagger} e_{\vec{m}}-\sum_{\vec{n}} \eta_{\vec{n} \vec{m}}^{v} \tilde{g}_{\vec{n}} e_{\vec{m}}\right) a_{\vec{k} v}^{\dagger}+\text { h.c. }
$$

where $a_{\vec{k} \nu}^{\dagger}\left(a_{\vec{k} \nu}\right)$ are creation (annihilation) operators of photons of wavenumber $\vec{k}$, polarization $\nu$, and frequency $\omega_{\nu}(\vec{k})$. In eq. (15), $\vec{d}$ is the atomic dipole, $\vec{\epsilon}_{k v}$ is the polarization vector, and $\rho(\vec{k})$ the density of states. The Frank-Condon (FC) factors $\eta_{0 \vec{m}}^{c}=\int d \vec{r} \psi_{\vec{m}}^{e}(\vec{r}) \exp (i \vec{k} \vec{r}) \psi_{0}(\vec{r})$ characterize the transitions into the condensate, whereas $\eta_{\vec{n} \vec{m}}^{u}=\int d \vec{r} \psi_{\vec{m}}^{e}(\vec{r}) \exp (i \vec{k} \vec{r}) u_{\vec{n}}(\vec{r})$ determine the transition into the $u$ quasiparticle wavefunctions. In the same way, the coefficients $\eta_{\vec{n} \vec{m}}^{v}$ characterize the transition into the $v$ wavefunctions. Finally, the vacuum energy is provided by the Hamiltonian: $\mathcal{H}_{f}=\sum_{\nu} \int \overrightarrow{d k} \omega_{\nu}(\vec{k}) a_{\vec{k} \nu}^{\dagger} a_{\vec{k} \nu}$. The physics of the system is therefore described by the Hamiltonian $\mathcal{H}=\mathcal{H}_{a}+\mathcal{H}_{a f}+\mathcal{H}_{f}$, where the atomic Hamiltonian can be written in the form (extracting the condensate energy) $\mathcal{H}_{a}=\sum_{\vec{n}} \tilde{\omega}_{\vec{n}} \tilde{g}_{\vec{n}}^{\dagger} \tilde{g}_{\vec{n}}+\sum_{\vec{m}}\left(\omega_{0}+\tilde{\omega}_{\vec{m}}^{e}\right) \tilde{e}_{\vec{m}}^{\dagger} \tilde{e}_{\vec{m}}$ where $\omega_{0}$ is the transition frequency between $|g\rangle$ and $|e\rangle$.

From the previous Hamiltonian, and using standard techniques of quantum stochastic processes [20, we get in Born-Markov approximation the QME for the density matrix $\rho$ :

$$
\dot{\rho}(t)=-i H_{e f f} \rho+i \rho H_{e f f}^{\dagger}+\mathcal{J} \rho
$$


where $H_{\text {eff }}$ is the effective (non-Hermitian) Hamiltonian, and

$$
\mathcal{J} \rho=\gamma\left\{\sum_{\vec{m}} \Re_{\vec{m} 00 \vec{m}}^{c} \tilde{g}_{0}^{\dagger} \tilde{e}_{\vec{m}} \rho \tilde{g}_{0} \tilde{e}_{\vec{m}}^{\dagger}+\sum_{\vec{m}, \vec{n}} \Re_{\vec{m} \vec{n} \vec{n} \vec{m}}^{u} \tilde{g}_{\vec{n}}^{\dagger} \tilde{e}_{\vec{m}} \rho \tilde{g}_{\vec{n}} \tilde{e}_{\vec{m}}^{\dagger}+\sum_{\vec{m}, \vec{n}} \Re_{\vec{m} \vec{n} \vec{n} \vec{m}}^{v} \tilde{g}_{\vec{n}} \tilde{e}_{\vec{m}} \rho \tilde{g}_{\vec{n}}^{\dagger} \tilde{e}_{\vec{m}}^{\dagger}\right\},
$$

is the jump super operator, where $\Re_{\vec{m} \vec{n} \vec{n}^{\prime} \vec{m}^{\prime}\left(\omega_{0}\right)}^{a}=\int d \Omega W(\Omega) \eta_{\vec{m} \vec{n}}^{a}\left(\omega_{0} \hat{e}_{k}\right) \eta_{\vec{m}^{\prime} \vec{n}^{\prime}}^{a}\left(\omega_{0} \hat{e}_{k}\right)^{*}(a=c, u$, $v)$, with $W(\Omega)$ denoting the emission pattern, and $\gamma$ is the spontaneous emission rate.

The effective Hamiltonian $H_{\text {eff }}$ is related with photon reabsorption processes, whereas the jump superoperator describes a spontaneous emission act without any further reabsorption. In this paper, we consider the reabsorption processes as negligible. As commented above, this should be the case if the Festina Lente condition is fulfilled [9], or alternatively for atoms with a three-level $\Lambda$ scheme under the conditions of ref. 12. Therefore, we constrain ourselves to the analysis of the jump processes from $|e\rangle$ to $|g\rangle$. From eq. (7) the amplitude for a jump from a state $\vec{n}_{e}$ of the $|e\rangle$ trap to a state $\vec{n}_{g}^{(a)}(a=u, v, c)$ of the $|g\rangle$ one is given by

$$
\Gamma_{\vec{n}_{g}^{(a)}, \vec{n}_{e}}=\gamma \int d \Omega W(\Omega)\left|\left\langle\vec{n}_{g}^{(a)}\left|e^{i \vec{k} \vec{r}}\right| \vec{n}_{e}\right\rangle\right|^{2},
$$

with $\left\langle\vec{r} \mid \vec{n}_{e}\right\rangle=R_{l_{e} n_{e}}(r) Y_{l m}(\Omega)$, and $\left\langle\vec{r} \mid \vec{n}_{g}^{(a)}\right\rangle=R_{l_{g} n_{g}}^{(a)}(r) Y_{l m}(\Omega)$. The FC factors are of the form

$$
\left\langle\vec{n}_{g}^{(a)}\left|e^{i \vec{k} \vec{r}}\right| \vec{n}_{e}\right\rangle=4 \pi \sum_{l^{\prime \prime}=0}^{\infty} \sum_{m=-l^{\prime \prime}}^{l^{\prime \prime}}(i)^{l^{\prime \prime}} Y_{l^{\prime \prime} m}^{*}\left(\Omega_{k}\right) A_{n_{g} l_{g} n_{e} l_{e}}^{l^{\prime \prime}(a)} B_{l_{g}}^{l^{\prime \prime} m_{g} l_{e} m_{e}}
$$

where $A_{n_{g} l_{g} n_{e} l_{e}}^{l^{\prime \prime}(a)}=\int d r r^{2} R_{l_{g} n_{g}}^{a}(r) j_{l^{\prime \prime}}(k r) R_{l_{e} n_{e}}(r)$ is the radial integral. The angular part is provided by $B_{l_{g} m_{g} l_{e} m_{e}}^{l^{\prime \prime}}=\int d \Omega_{r} Y_{l_{g} m_{g}}^{*}\left(\Omega_{r}\right) Y_{l^{\prime \prime} m}\left(\Omega_{r}\right) Y_{l_{e} m_{e}}\left(\Omega_{r}\right)$, solvable in terms of the corresponding 3-j symbols. Due to the properties of these symbols, $B_{l_{g} m_{g} l_{e} m_{e}}$ is independent of $m$ since $-m_{g}+m_{e}+m=0, l_{g}+l_{e}+l^{\prime \prime}=2 p$ (where $p$ is an integer), and $\left|l_{g}-l_{e}^{\prime}\right| \leq l^{\prime \prime} \leq l_{g}+l_{e}$. From the amplitudes (8) and the populations of the corresponding levels, we obtain the transition probabilities describing creation of a quasiparticle $n=\left(n_{g}, l_{g}\right)$

$$
P_{n}^{u}=\left(\left\langle N_{n}(T)\right\rangle+1\right) \sum_{n_{e} l_{e}}\left\langle N_{\vec{n}_{e}}\left(T_{e}\right)\right\rangle \sum_{m_{g}, m_{e}} \Gamma_{\vec{n}_{g}^{(u)}, \vec{n}_{e}} .
$$

Similar expressions are obtained for the transition probability of destruction of a quasiparticle $n$ associated with the transfer of a particle into the condensate after thermalization, $P_{n}^{v}$, and the direct decay process into the condensate, $P^{c}$. In eq. (10), $\left\langle N_{n}(T)\right\rangle=\left(\exp \left[E_{n} / K_{B} T\right]-1\right)^{-1}$ is the Bose-Einstein distribution in the $|g\rangle$ trap, and $\left\langle N_{\vec{n}_{e}}\left(T_{e}\right)\right\rangle=\hat{N} \exp \left[-E_{\vec{n}_{e}} / K_{B} T_{e}\right]$ is the Boltzmann distribution (normalized to 1 ) in the $|e\rangle$ trap.

Knowing the transition probabilities $P_{n}^{u}$ (10), $P_{n}^{v}$ and $P^{c}$ we can simulate the effects of a spontaneous emission process. We assume that the time scale of thermalization in the $|g\rangle$ trap is much faster than the time interval between different pump events, i.e. the collisional rate in the ground-state trap is much larger than $\gamma$. In this way, after every pump, the system re-thermalizes, redistributing the energy gained or lost during the pump process. We analyze in the following the variation of the condensate temperature after the pumping.

After pumping, the number of $|g\rangle$ atoms increases to $N^{\prime}=N+1$, and the energy becomes:

$$
\left\langle E^{\prime}\right\rangle=E+\mu P^{c}+\sum_{n}\left(\epsilon_{n}+\mu C_{n}\left(P_{n}^{u}-P_{n}^{v}\right) .\right.
$$


where $E$ is the energy before the pumping, and $C_{n}=\left|u_{n}\right|^{2}+\left|v_{n}\right|^{2}$. After the re-thermalization, the system acquires a new temperature $T^{\prime}$ which can be evaluated from the expressions:

$$
\begin{aligned}
E^{\prime} & =\left\langle\left.\psi_{0}\left|\mathcal{H}_{G}-\frac{U_{g g}}{2}\right| \psi_{0}\right|^{2} \mid \psi_{0}\right\rangle+\sum_{n} N_{n}\left(T^{\prime}\right)\left(\epsilon_{n}+\mu C_{n}\right)-\sum_{n}\left(\epsilon_{n}-\mu\right)\left|v_{n}\right|^{2}, \\
N_{0}\left(T^{\prime}\right) & =N+1-\sum_{n} N_{n}\left(T^{\prime}\right) C_{n}+\left|v_{n}\right|^{2},
\end{aligned}
$$

where $N_{n}(T)=\left(\exp \left[\epsilon_{n} / K_{B} T\right]-1\right)^{-1}$ is the Bose-Einstein distribution in the $|g\rangle$ trap. In (12) and (13), we assume the excitation spectrum as that calculated for $N$ particles, i.e. before the pumping. This approximation is valid as long as the number of excited particles $N-N_{0} \gg 1$.

Assuming $T^{\prime}=T+\delta T$ with $\delta T \ll T$, we can perform a Taylor expansion up to first order in $\delta T / T$. Substituting into eqs. (12) and (13), we can obtain the expression for $\xi=$ $\left(N_{0}^{\prime} / N^{\prime}-N_{0} / N\right) /\left(N_{0} / N\right)$. If $\xi>0$, the pumping increases the relative population in the condensate, and therefore the temperature of the sample is reduced. On the contrary, if $\xi<0$ the system is heated by the pumping. It is easy to see that up to terms of the order $\delta N / N_{0}^{2}$, where $\delta N=N-N_{0}$, the system is cooled $(\xi>0)$ when the condition:

$$
\left(N_{0}-1\right) \frac{\tilde{\mu}\left(N_{0}\right)\left(1-P^{c}\right)-\sum_{n}\left(\tilde{\epsilon}_{n}+\tilde{\mu} C_{n}\right)\left(P_{n}^{u}-P_{n}^{v}\right)}{\sum_{n} \tilde{\epsilon}_{n}^{2} \frac{\exp \left[\tilde{\epsilon}_{n}\right]}{\left(\exp \left[\tilde{\epsilon}_{n}\right]-1\right)^{2}}} \sum_{n} \tilde{\epsilon}_{n} C_{n} \frac{\exp \left[\tilde{\epsilon}_{n}\right]}{\left(\exp \left[\tilde{\epsilon}_{n}\right]-1\right)^{2}}>\delta N
$$

is fulfilled, with $\tilde{\mu}=\mu / K_{B} T, \tilde{\epsilon_{n}}=\epsilon_{n} / K_{B} T$. For moderately low $T$ this condition is typically fulfilled. For large $T, \delta N$ can increase enough to violate (14). On the other hand for very low $T$ the transition probability into the condensate $P^{c} \rightarrow 1$, and also in this case the condensate is heated. Hence, there is a transition from heating to cooling at some finite threshold temperature, $T_{t h}$, which depends on four different parameters: the total number of atoms $N$, the temperature, $T_{e}$, of the excited state trap, the coupling constant $U_{e g}$ for the collisions between $|e\rangle$ and $|g\rangle$ atoms, and the ratio $\omega_{e} / \omega_{g}$ between the frequencies of the $|g\rangle$ and $|e\rangle$ traps. When $N$ increases, so does $\tilde{\mu}$ and it is easy to see that $T_{t h}$ decreases. If $T_{e}$ increases the transition to higher lying excited states are more favorable, and as one could expect $T_{t h}$ increases. The parameters $U_{e g}$ and $\omega_{e} / \omega_{g}$ influence the spectrum of the excited state trap. For $U_{e g}=0$ the harmonic oscillator spectrum is recovered. However, when $U_{e g}$ grows, the mean-field repulsion leads to an expulsion of the $|e\rangle$ atoms from the trap center, increasing the probability for a transition into excited states of the $|g\rangle$ trap. On the other hand, if $\omega_{e} \gg \omega_{g}$, and $T_{e}$ is sufficiently small, the mean-field shift just provides an overall homogeneous shift of the levels of the $|e\rangle$ trap, and the $T_{t h}$ becomes comparable to that for $U_{e g}=0$.

We have analyzed $\delta T$ after a pumping using the following procedure. For given values of $N$ and $T$, we first calculate the number of atoms in the BEC, $N_{0}$, and evaluate $\psi_{0}$ by evolving eq. (11) in imaginary time. Then, we diagonalize eqs. (2) and (31) employing a harmonic oscillator basis, to find the $\{u, v\}$ eigenfunctions, and the corresponding eigenenergies $\left(^{1}\right)$. As a next step, we calculate $\Gamma_{\vec{n}_{g}^{(a)}, \vec{n}_{e}}(a=c, u, v)$ using eq. (8). For a given $T_{e}$ we evaluate $P_{n l}^{u}, P_{n l}^{v}$ and $P^{c}$. From eq. (11) we obtain the new average energy after the pumping. Finally, from eqs. (12) and (13) we obtain the new temperature after the rethermalization.

Fig. 17 shows the variation of $T / T_{c}$ after a spontaneous emission as a function of the initial $T / T_{c}$ for $\omega=20 \times 2 \pi \mathrm{Hz}, \omega_{e}=10 \omega_{g}, T_{e}=21 \hbar \omega_{g}$, and $N=10^{4}, 5 \times 10^{4}$ and $10^{5}$. In the calculation of the FC factors (9), we have considered a Lamb-Dicke parameter

$\left({ }^{1}\right)$ In TF regime $u_{n}, v_{n}$ and $\epsilon_{n}$ are known analytically. We employ here however the numerical approach, which works in all intermediate regime between weakly condensed and TF regime 

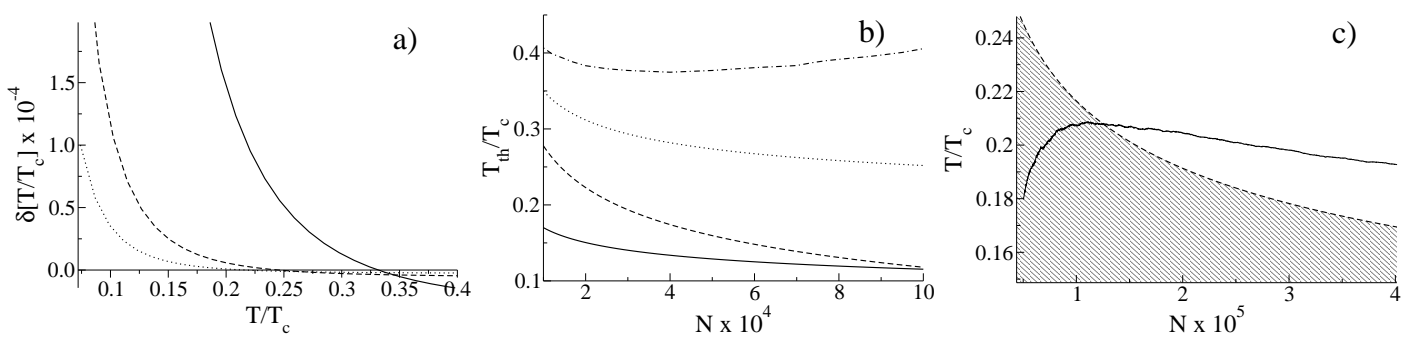

Fig. 1 - a) variation of $T / T_{c}$ after a spontaneous emission as a function of the initial $T / T_{c}$ for $\omega=20 \times 2 \pi \mathrm{Hz}, \omega_{e}=10 \omega_{g}, T_{e}=21 \hbar \omega_{g}$, and $N=10^{4}, 5 \times 10^{4}$ and $\left.10^{5} ; \mathrm{b}\right)$ dependence of $T_{t h}$ on $N$ for $\omega_{e}=\omega_{g}$ and $U_{e g} / U_{g g}=1$ (dotted-dashed curve), 0.5 (dotted curve), and 0 (dashed curve); the solid curve shows the case $\omega_{e}=10 \omega_{g}$ and $U_{e g} / U_{g g}=1$; c) solid line indicates the Monte Carlo simulation of the loading process and dashed line $T_{t h}$ for $\omega_{e}=10 \omega_{g}, T_{e}=21 \hbar \omega_{g}$

$\eta^{2} \equiv E_{r e c} / \hbar \omega_{g}=1$, where $E_{r e c}$ is the photon recoil energy. The choice of $\eta$ and $T_{e}$ is due to our numerical limitations, but we expect qualitatively similar results for other values of these parameters. As previously discussed, the system is cooled for $T>T_{t h}$, where $T_{t h}$ is progressively lower for larger $N$. In fig. 1h, due to the low value of $T_{e}$ chosen, it is not possible to observe the heating region for large temperatures, which for this case is located at $T$ close to $T_{c}$. For other ranges of parameters the qualitative picture does not change significantly, i.e. always a threshold temperature separating heating and cooling regions exists.

We have analyzed the dependence of $T_{t h}$ on different parameters. Fig. 10 shows the dependence of $T_{t h}$ on $N$ for $\omega_{e}=\omega_{g}$ and different values of $U_{e g}$. For $U_{e g} \rightarrow 0, T_{t h}$ decays monotonically with $N$. However, when $U_{e g}$ increases, and due to the repulsive mean-field induced by the $e-g$ collisions, the eigenfunctions of the $|e\rangle$ trap possess a local minimum at the trap center, and the pumping into the condensate becomes less effective. Consequently, $T_{t h}$ increases for large $N$, as observed for $U_{e g}=U_{g g}$. This effect is less pronounced, for larger values of $\omega_{e}$ keeping fixed $T_{e}$, since then the lower levels of the $|e\rangle$ trap have an extension smaller than the BEC wavefunction, and hence the mean-field just produces a global energy shift of the lowest $|e\rangle$ levels, with no consequences for the pumping (see fig. 1b).

Finally, we have simulated for different conditions the continuous optical pumping into the BEC. To this aim, we have employed Monte Carlo methods to evaluate the corresponding rate equations, with transition probabilities $P_{n l}^{u}$ (10), $P_{n l}^{v}$ and $P^{c}$. In principle, after every pumping the previously described algorithm should be repeated. In practice, it is enough to do so after a number of pumps $N_{\text {pumps }} \ll \delta N$, which is dynamically adjusted in our calculations. Fig. 15: shows our results for $\omega_{e}=10 \omega_{g}, T_{e}=21 \hbar \omega_{g}$. The dashed curve in fig. 11 indicates $T_{t h}$. The simulation is started within the heating region (below the $T_{t h}$ curve), with $N=4.8 \times 10^{4}$, and $T / T_{c}=0.18$. As expected, $T$ increases with the pumping. However, once $\left(N, T / T_{c}\right)$ crosses the $T_{t h}$ curve, the temperature begins to decrease when pumping. Therefore, interestingly, the temperature of the system should asymptotically approach the curve $T_{t h}$.

In this paper, we have analyzed the continuous optical loading of a BEC in the ThomasFermi regime. Contrary to the weak-condensation case, the BEC and excited-state wavefunctions vary during the loading. By means of GPE and Bogoliubov equations, we have determined before every pumping the proper wavefunctions, and transition probabilities. Assuming rapid thermalization, we have monitored the variation of the temperature during the loading. We have observed that for a given number of trapped atoms, $N$, there is always a threshold temperature, $T_{t h}$, below which the BEC is heated. The concept of $T_{t h}$ is very general and should also apply to collisional loading of a BEC. We have analyzed the dependence of $T_{t h}$ 
on different parameters, in particular $N$, the trap geometries, and the interparticle interactions. A lower $T_{t h}$ is obtained for smaller interactions between excited-state and ground-state atoms, since repulsive interactions displace the excited-state atoms away from the trap center, preventing the effective pumping into the BEC. Our analysis shows that, not only the number of atoms in the trap, but also the temperature of the system, can be maintained by means of optical loading. To this aim, the condensate should be created with a temperature and a number of atoms, lying close to the curve $T_{t h}(N)$. In that case, in the presence of losses, if the loss mechanism leads to heating, $T$ becomes larger than $T_{t h}(N)$, and the system is cooled by next pumpings, whereas the opposite is true if the loss mechanism cools. Interestingly, this could allow for the sustained analysis of BEC at a quasi-constant temperature.

Several physical scenarios could be devised for the continuous optical loading of a BEC. For example, an optical lattice could be used to move atoms in an internal state $|r\rangle$ from a relatively hot reservoir into the center of the BEC region, where a Raman pulse could be employed to transfer the $|r\rangle$ atoms into an state $|e\rangle$, which decays into the ground-state $|g\rangle$. In this way, it could be achieved that only those lattice sites near the condensate center actually decay into the $|g\rangle$ trap. For a sufficiently strong optical lattice each lattice site will behave as a single trap with few occupied levels, as that discussed in our paper. Interestingly, in this scenario, due to the small size of the $|e\rangle \operatorname{trap}, T_{t h}$ should monotonically decay with $N$, since the mean-field effects should just shift globally the states of the $|e\rangle$ trap. Therefore, in this system the pumping could be employed to post-cool an already formed BEC down to $T=0$, and maintain the condensate temperature very low against possible loss sources.

We acknowledge support from the Alexander von Humboldt Stiftung, the Deutsche Forschungsgemeinschaft, EU RTN Network "Cold Quantum Gases", ESF PESC Program BEC2000+.

\section{REFERENCES}

[1] Inguscio M., Stringari S. and Wieman C. (Editors), Proceedings of the International School of Physics "Enrico Fermi", Vol. Course CXL, (IOS Press, Amsterdam) 1999.

[2] See e.g., Meystre P., Atom Optics (Springer Verlag) 2001

[3] Mewes M. O. et al., Phys. Rev. Lett., 78 (1997) 582.

[4] Bloch I., Hänsch Th., And Esslinger T., Science, 403 (2000) 166.

[5] Hagley E. W. et al., Science, 283 (1999) 1706.

[6] Andrews M. R. et al., Science, 275 (1997) 637.

[7] Anderson B. P. And Kasevich M.A., Science, 282 (1998) 1686.

[8] Chikkatur A. P. et al., Science, 296 (2002) 2193.

[9] Mandonnet E. et al., Euro. Phys. J. D, 10 (1999) 9.

[10] Roos C.F. et al., Europhys. Lett., 61 (2003) 187.

[11] Spreeuw R. J. C. et al., Europhys. Lett., 32 (1995) 469; Janicke U. and Wilkens M., Adv. At. Mol. Opt. Phys., 41 (1999) 261.

[12] Santos L. et al., Phys. Rev. A, 63 (2001) 063408.

[13] Stuhler J. et al., Phys. Rev. A, 64 (2001) 031405.

[14] Olshan'it M., Castin Y. and Dalibard J., Proc. $12^{\text {th }}$ Int. Conf. on Laser Spectroscopy, edited by M. Inguscio, M. Allegrini, and A. Lasso (World Scientific, Singapour) 1996

[15] Cirac J. I., Lewenstein M. and Zoller P., Europhys. Lett., 35 (1996) 647.

[16] Cirac. J. I. and Lewenstein M., Phys. Rev. A, 53 (1996) 2466.

[17] Floegel F., Santos L. and Lewenstein M., Euro. Phys. Lett., 54 (2001) 279.

[18] Santos L. and Lewenstein M., Appl. Phys. B, 69 (1999) 363.

[19] Dalfovo F. et al., Rev. Mod. Phys., 71 (1999) 463.

[20] See e.g., Gardiner. C, Quantum Noise (Springer Verlag, Berlin) 1991.

[21] Castin Y. and Dum R., Phys. Rev. A, 57 (1998) 3008. 